\title{
Multiview plus depth video using high efficiency video coding method
}

\begin{abstract}
The main problem associated with 3D video delivery is huge data transmission rate especially when the data to be submitted are large video files such as multiview plus depth videos. This paper describes a new method of compression for multiview videos with depth data by using the new High Efficiency Video Coding (HEVC) technology. We propose a new compression method by applying the Reduced Resolution Depth Coding (RRDC) method to the depth videos. RRDC is applied by Down-Sampling and Up-Sampling (DSUS) the depth data of the multiview videos. The depth data is down-sampled before HEVC encoding and up-sampled after HEVC decoding operation. The proposed depth compression method used with HEVC showed a $20 \%$ savings at low bit rate when tested with 2 views plus depth video sequence.
\end{abstract}

